\title{
Combination of hardening mechanisms in crystalline materials
}

\author{
François Louchet $\left({ }^{1}\right)$, Abdelhafid Triki $\left({ }^{1}\right)\left({ }^{\star}\right)$ and Joseph Pelissier $\left({ }^{2}\right)$ \\ ( $\left.{ }^{1}\right)$ LTPCM, ENSEEG, INP de Grenoble, BP 75, 38402 st Martin d'Heres, France \\ (2) CEA-CEREM, Centre d'Etudes Nucléaires de Grenoble, BP 85X, 38042 Grenoble Cedex, France
}

(Received January 18, 1993; accepted April 23, 1993)

\begin{abstract}
Résumé . - Deux cas de combinaison entre durcissement par obstacles localisés et par friction de réseau sont étudiés par déformation in situ. Le Germanium illustre un cas de durcissement par la forêt dans lequel la friction résiduelle sur les décrochements modifie de façon significative le mécanisme de franchissement. Dans le cas des alliages $\mathrm{Fe} \mathrm{Cr}$, le durcissement par décomposition spinodale entraîne une localisation de la déformation et accentue le mouvement sautillant des vis.
\end{abstract}

\begin{abstract}
Two cases of combination of hardening by localized obstacles and hardening by lattice friction are studied by in situ straining experiments. The case of forest obstacles is illustrated by Germanium, where residual friction on kinks modifies significantly the overcoming mechanism. In the case of $\mathrm{Fe} \mathrm{Cr}$ alloys, hardening by spinodal decomposition yields strain localization and enhances jerky motion of screws.
\end{abstract}

\section{Introduction.}

In this paper, in situ experiments are used to investigate the coupling between lattice friction and two other types of obstacles to dislocation motion: forest hardening and precipitation hardening. In the first case (Germanium), the influence of forest obstacles on kink motion is examined while Peierls resistance is varied continuously by increasing temperature. In the second case (concentrated $\mathrm{Fe}$ Cr alloys), the intrinsic lattice friction characteristic of the BCC structure is combined to an increasing precipitation hardening due to the spinodal decomposition of the disordered alloy, which results in a spatial modulation of $\mathrm{Cr}$ concentration.

\section{Lattice friction and forest hardening: Germanium.}

In order to vary the Peierls frictional stress, a single crystal of Ge has been strained in a HVEM operating at $400 \mathrm{kV}$, at temperatures ranging from $450^{\circ} \mathrm{C}$ up to the melting point.

$\left(^{\star}\right)$ now at: Institut de Physique, Université d’Annaba, BP 12, Annaba, Algeria. 
In the low temperature domain, $\left(T=400^{\circ} \mathrm{C}\right)$, lattice friction on screws and $60^{\circ}$ dislocations prevails. A previous work [1] has shown that, depending on dislocation length, a continuous change between the two asymptotic velocity regimes predicted by Hirth and Lothe [2] can be evidenced: the velocity of very short dislocations is proportional to their lengths $L$, whereas that of much longer ones is independent of $L$. From these in situ observations, activation energies for double kink nucleation and kink migration have been derived, giving an estimate of the kink formation energy (about $0.5 \mathrm{eV}$ ) and of the kink migration energy (about $0.9 \mathrm{eV}$ for $60^{\circ}$ dislocations and about 0.75 to $0.8 \mathrm{eV}$ for screws). The typical dislocation length separating these two limiting regimes is the mean free path of kinks $X$.

The influence of forest obstacles on dislocation velocities is different in the two regimes:

For large forest densities, i.e. short dislocations ( $L<X=0.3$ to $0.5 \mu \mathrm{m}$ in usual conditions), forest obstacles influence significantly dislocation mobilities since they determine the length on which double kinks can be nucleated in a competitive way. The larger the forest density, the shorter is the free dislocation length $L$, and the slower the dislocation. This mechanism is also at the basis of hardening of BCC metals at low temperatures, but in a wide range of forest densities, since in this case kink motion is much easier and results in a very large value of $X$. The dislocation velocity is given by [2]:

$$
v=\nu_{\mathrm{D}}\left(\tau b^{3} / k T\right) L \exp \left(-\left(F^{*}+W_{\mathrm{m}}\right) / k T\right)
$$

where $v_{\mathrm{D}}$ is the Debye frequency, $F^{*}$ the saddle point energy corresponding to the critical double kink configuration, $\tau$ the resolved shear stress and $W_{\mathrm{m}}$ the migration energy of kinks. As demonstrated in the next paragraph, forest overcoming is not thermally activated here, and $L$ can be determined by a steady state argument [3] to be:

$$
L=\tau /\left(\alpha \mu b \rho_{\mathrm{f}}\right)
$$

where $\rho_{\mathrm{f}}$ is the forest density and $\alpha$ a geometrical factor close to 1 which accounts for the obstacle strength. From equations (1) and (2), the dislocation velocity is related to the forest density by:

$$
v=\nu_{\mathrm{D}}\left(\tau^{2} b^{2} / \alpha \mu \rho_{\mathrm{f}} k T\right) \exp \left(-\left(F^{*}+W_{\mathrm{m}}\right) / k T\right)
$$

For small forest densities, which is usually the case in semiconducting materials, the velocity of long dislocations $(L>X)$ is controlled by the mean free path of kinks $X$. It becomes insensitive to the forest, and is given by the usual equation (2):

$$
v=\nu_{\mathrm{D}}\left(\tau b^{3} / k T\right) b \exp \left(-\left(F^{*}+2 W_{\mathrm{m}}\right) / 2 k T\right)
$$

In both cases anyway, plasticity is controlled by lattice friction, the role of localized obstacles being to modify the kinetics of the double kink mechanism for forest densities larger than a critical value.

As temperature is increased, Peierls relief is smoothed out, and elastic effects (mainly dislocation interactions) become apparently prominent as compared to core effects. Dislocation motion, which was governed by viscous flow of individual dislocations, becomes progressively collective. Plastic flow is now controlled by local dislocation interactions (junction formation (Fig. 1), repulsive cutting,...), whose overcoming kinetics can be helped by collective effects (pile-ups). The local stress under which obstacles are overcome can be quite different of the applied stress, and can be measured directly during in situ experiments [4]. In the case of repulsive interactions, both the waiting time $\Delta t$ and the local stress $\tau$ have been estimated from in situ recordings. The activation energy $\Delta G$ for repulsive forest cutting can be directly estimated in principle by:

$$
\Delta G=k T \ln \left[\nu_{\mathrm{D}}(b / l) \Delta t\right]
$$



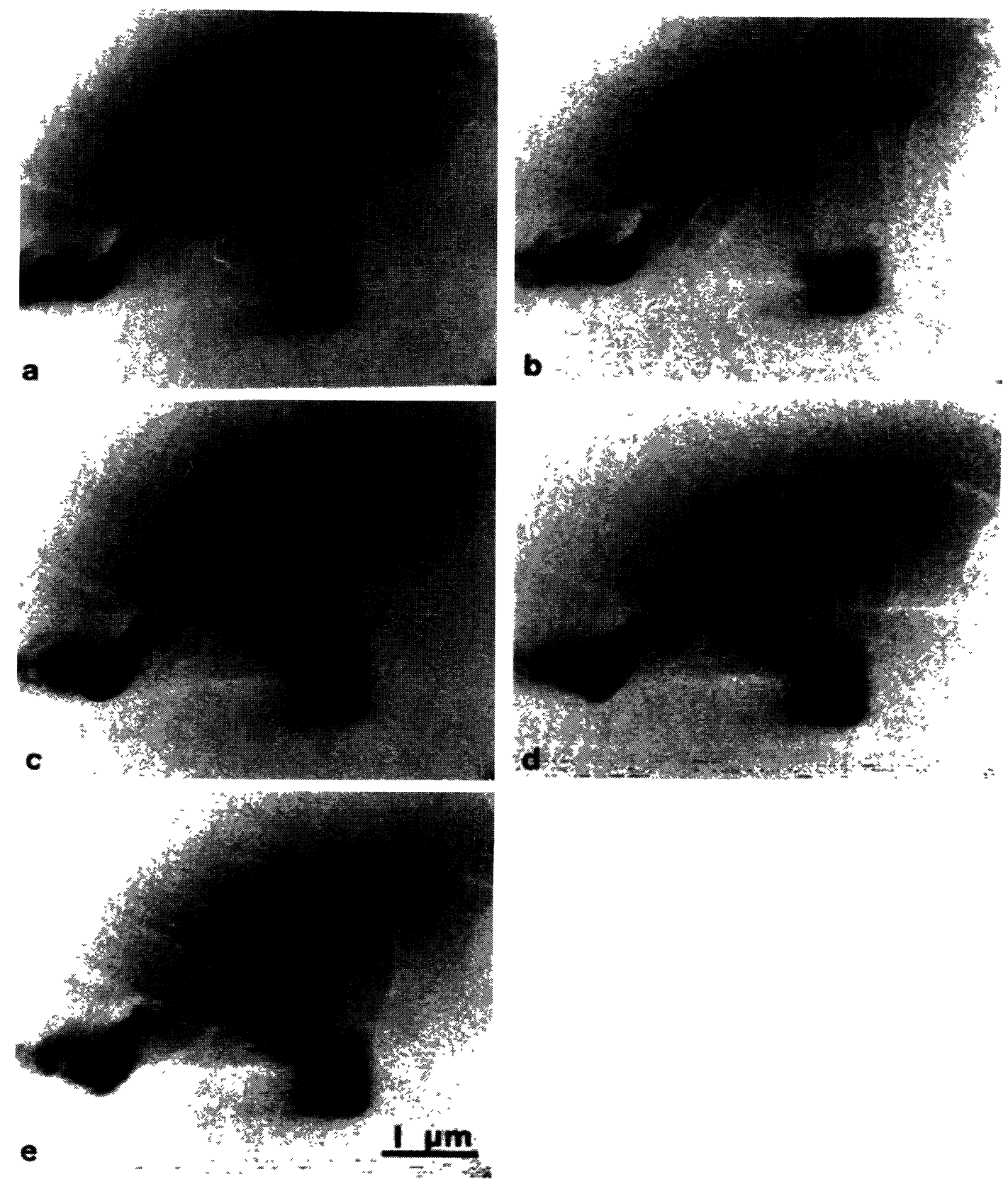

Fig. 1. - Formation and destruction of an attractive junction in Ge (about $10 \mathrm{~s}$ between a and e), $T=$ $790{ }^{\circ} \mathrm{C}$.

where $\nu_{\mathrm{D}}$ is the Debye frequency, $b$ the modulus of the Burgers vector, and $l$ the length of the dislocation. Typical values are $\Delta t=1.5$ to $8 \mathrm{~s}, l=0.7 \mu \mathrm{m}, \tau=70 \mathrm{MPa}, T=550$ to $700{ }^{\circ} \mathrm{C}$, 
which should lead to activation energies $\Delta G$ of about $1.8 \mathrm{eV}$. Since $\Delta G=\Delta G(\tau)=\Delta G$ o $l b^{2} \tau$, the height of the obstacle $\Delta G$ o can be estimated. Surprisingly the stress contribution $l b^{2} \tau$ is found unreasonably high $(50 \mathrm{eV})$ and should give tremendous values of $\Delta G$ o. This probably means that kink migration energy, which is much larger than in metals, inhibits the vibration of the whole dislocation segment of length $l$, even at very high temperatures $\left(T / T_{\mathrm{m}} \cong 0.8\right.$ or 0.9). Comparing the characteristic frequencies for dislocation vibration $\left(\nu_{\mathrm{D}} b / l\right)$ and for kink jump $\nu_{\mathrm{D}} \exp \left(-W_{\mathrm{m}} / k T\right)$, one can get a typical length $l_{0}=b \exp \left(W_{\mathrm{m}} / k T\right)$. For dislocation lengths $l>l_{0}$, the vibration frequency is smaller than the jump frequency of kinks, and kink motion does not hinder the vibration of the dislocation. For short segments $(l<l o)$, kink drift is rate controlling. Introducing the above typical figures, one gets values of $l_{0}$ ranging between 6 to $30 \mu \mathrm{m}$ when temperature is decreased from $700{ }^{\circ} \mathrm{C}$ to $550{ }^{\circ} \mathrm{C}$. This characteristic length is much larger than the observed spacings between forest obstacles $(l=0.7 \mu \mathrm{m})$. It can be reasonably thought that forest overcoming is not helped here by thermal activation, since it needs at least the formation of two jogs of energy (5): $2 U j \cong \mu b^{3} / 10 \cong 5 \mathrm{eV}$, and the small activation volume (about $b^{3}$ ) should make the external stress quite inefficient in a thermally activated mechanism, which would take indeed geological times (at least $3 \times 10^{5}$ years). The overcoming should then be of a pure stress type, occurring when the cusp on the glide dislocation reaches a critical angle.

In spite of a qualitative similarity, the behaviours of standard FCC metals and of covalent diamond-type crystals (including covalent ceramics) even at very high temperatures are then fundamentally different, due to the need of breaking covalent bonds to move kinks.

Cross-slip is frequently observed (Fig. 2) as soon as dislocation glide becomes difficult (forest pinning, pile-up on strong obstacles...). However, it is remarkable that dislocation glide remains the easiest deformation mode up to melting point as compared to climb. Furthermore, though cross-slip of individual dislocations happens quite frequently, long high-density pile-ups are observed at temperatures very close to the melting point (Fig. 3). The reasons for this collective behaviour are not clear, although a slip localization by foil thinning cannot be excluded.

\section{Lattice friction and precipitation hardening: $\mathrm{Fe} \mathrm{Cr}$ alloys.}

The so-called $450^{\circ} \mathrm{C}$ hardening in concentrated $\mathrm{Fe} \mathrm{Cr}$ alloys is a complex problem involving both a strong Peierls stress typical of the BCC structure and a "precipitate" hardening by a high volume fraction of a "coherent" phase, arising from a spinodal decomposition of the as-quenched solid solution. Ordering increases as precipitation proceeds, which leads to pseudo-periodic spatial modulations of $\mathrm{Cr}$ concentration. This can be described as a precipitation with diffuse interfaces. The physical basis of the related hardening is likely to be a disordering, due to shearing by dislocations, of the "precipitates", i.e. of the concentration modulations of the spinodally decomposed alloy [6], which locally drives the alloy back to the initial disordered state, and then increases its free energy $G$. In the frame of this assumption, the resistant force on dislocations due to precipitate shearing is given by the derivative $\mathrm{d} G / \mathrm{d} x$ of the driving force $G$ for precipitation with respect to the dislocation displacement $x$. It seems reasonable to assess that this force is mainly experienced during kink motion rather during double-kink nucleation, since double-kink nucleation should occur preferentially in zones where the disorder induced by shear is a minimum, i.e. in zones where $\mathrm{Cr}$ concentration is an extremum. The screw velocity is then still controlled by a double kink mechanism, but on dislocation lengths which decrease as the spinodal decomposition proceeds since the mean free path of kinks is supposed to decrease [3]. The principles of coupling between lattice friction and precipitate hardening are somewhat similar to the above case of Peierls vs forest coupling. However, the shearing of the precipitated structure by dislocations is also expected to give rise to local softening and slip localization, which is actually observed in situ 

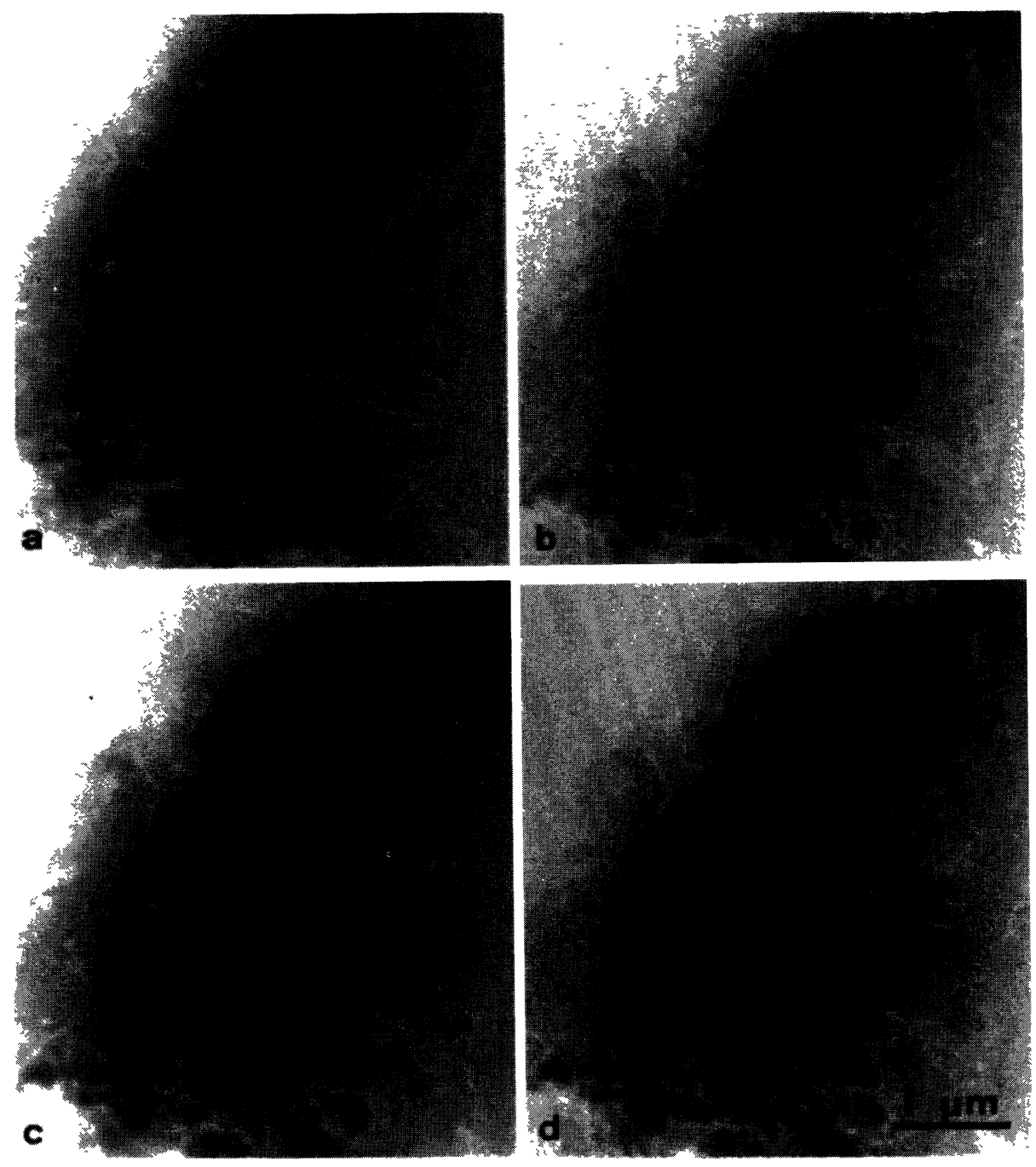

Fig. 2. - Cross-slip in $\mathrm{Ge}, T=610^{\circ} \mathrm{C}$.

(Fig. 4).

In addition, in situ experiments show that, in the hardened state, dislocations move in a more jerky way as compared to the disordered state. It is interesting to compare this last point with the behaviour of dislocations in other materials exhibiting a Peierls-type lattice friction.

Static TEM observations of materials exhibiting a strong Peierls stress show straight dislocations either along screw directions (BCC, HCP, intermetallics) or along screw and $60^{\circ}$ directions (diamond cubic (DC)). However, in situ experiments show that the motion of dislocations can be significantly different, depending on the material, or even on loading conditions in a given material. In DC crystals, dislocations always move smoothly. In BCC, HCP and intermetallics, screw dislocation motion can be either smooth or jerky, depending on loading conditions. In this latter case, the common screw character of the involved dislocations suggests that lattice friction is re- 


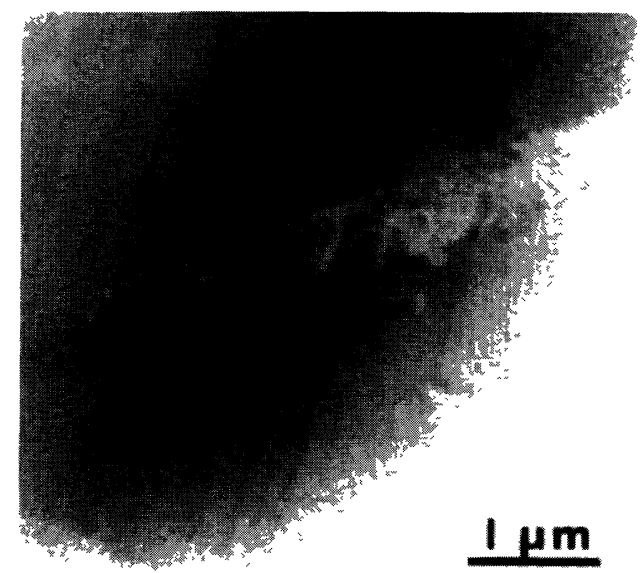

Fig. 3. - Highly localized slip close to the melting point in $\mathrm{Ge}: T=780{ }^{\circ} \mathrm{C}, T / T_{\mathrm{m}} \cong 0.9$.

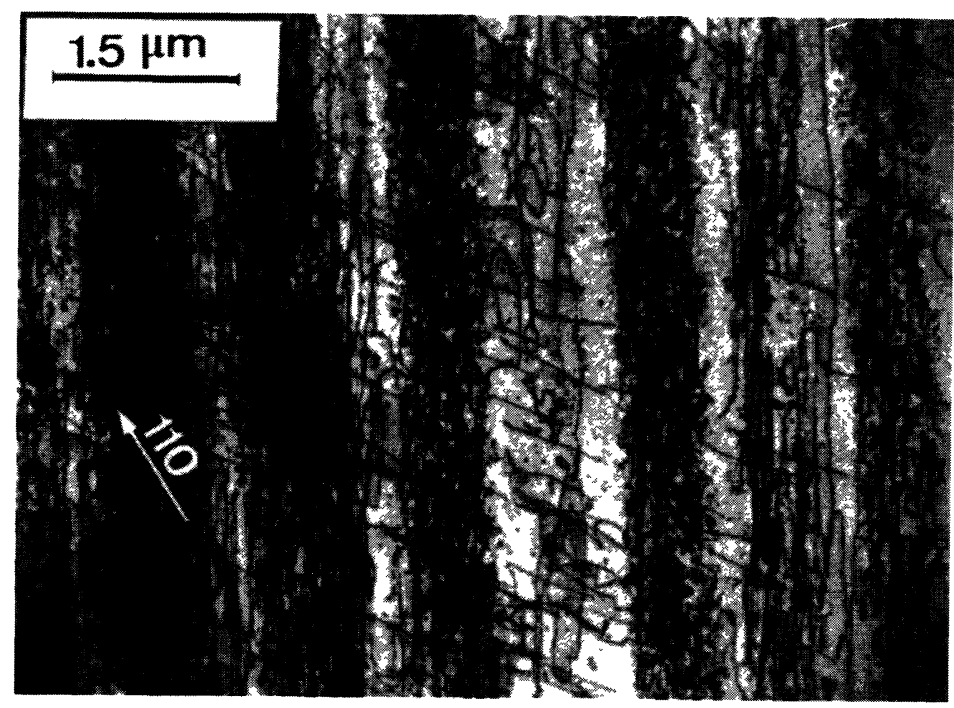

Fig. 4. - Slip localization due to local softening in Fe 35\% $\mathrm{Cr}$ anne.aled 505 hours at $T=500{ }^{\circ} \mathrm{C}$ and strained at room temperature. Dislocation movement is essentially jerky.

lated to cross-slip, whereas in the case of DC crystals the origin of the Peierls stress is obviously related to the specific core structure of the dislocations (covalent bonding). The jerky behaviour of screw dislocations in HCP and intermetallics has been interpreted in terms of a locking-unlocking mechanism [7], governed by cross-slip on resp. the basal or the cube slip planes. This type of mechanism is not possible in covalent crystals and this is an obvious reason for smooth motion of dislocations in this case. However, the question is still open in the case of BCC metals, in which the threefold nonplanar dissociation of screw dislocations is responsible for the lattice friction. There is up to now no theoretical evidence for the existence of a metastable glissile core con- 
figuration. In addition, in situ observations of jerky motion in annealed $\mathrm{Fe} \mathrm{Cr}$ alloys show that slip traces might be significantly curved between two neighbour locking sites (Fig. 5). This means that cross-slip occurs continuously during the fast jump of the dislocation, and then that the double kink mechanism operates at every atomic row between the locking positions. The existence of a glissile metastable state is therefore highly questionable in this case. Alternatively, elastic interactions between parallel gliding dislocations (enhanced by localization) could be invoked. Dislocation slowing down by cross-slip and subsequent formation of jogs on screws can also be an alternative (or complementary) mechanism for locking. In this case, unlocking could occur by sweeping out of these jogs by a superkink. In a thin foil, such superkinks can be produced at surfaces. In the bulk, they could arise from the fact that the different segments of a single screw have different lengths, and then different velocities. This question is of crucial importance in understanding the mechanical response of these materials, and in situ straining experiments are in this case an unavoidable means of investigation.

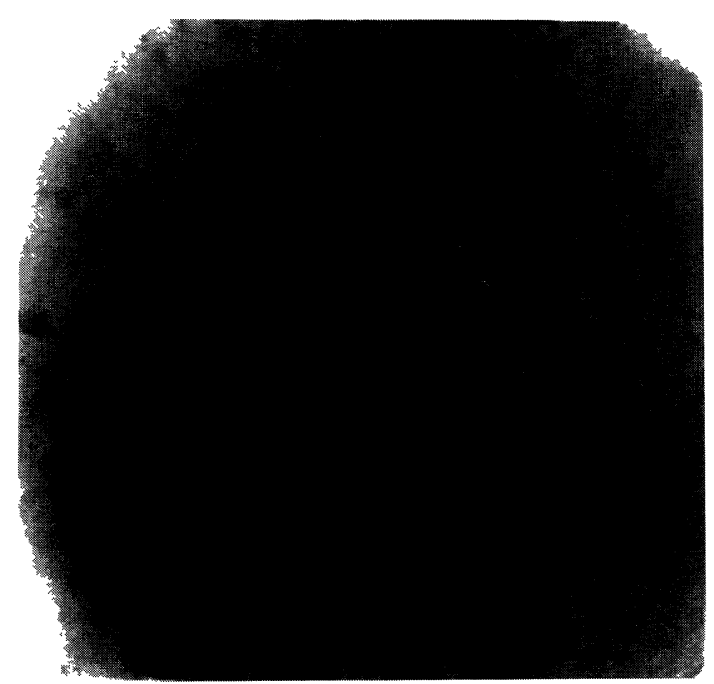

Fig. 5. - Jerky motion of a dislocation in $\mathrm{Fe} \mathrm{Cr}$ annealed $500 \mathrm{~h}$ at $500{ }^{\circ} \mathrm{C}$ : the curved shapes of slip traces gives evidence for continuous cross-slip during a fast jump of a dislocation. The final locking position is indicated by an arrow.

\section{Conclusion.}

In this work, in situ straining experiments have been used to investigate the combined effects of lattice friction and localized obstacles on dislocation motion. In the case of Germanium at low temperatures, which illustrates the case of a strong Peierls stress combined with forest obstacles, the velocity is always controlled by double kink nucleation and kink migration. The only role of forest obstacles is to reduce the prefactor through a reduction of the dislocation length, if the obstacle separation is smaller than the mean free path of kinks. At high temperatures, dislocation dynamics seem qualitatively similar to that in FCC metals. However, overcoming of repulsive 
forest obstacles is not thermally activated, since kink drift kinetics hinders vibrations of short dislocation segments, and repulsive forest must be overcome by stress only. In the case of concentrated $\mathrm{Fe} \mathrm{Cr}$ alloys hardened by spinodal decomposition, disordering of the concentration modulations by shearing hinders kink motion and is thought to be the dominant contribution to hardening. Screws are observed to move in a jerky way, but the existence of a metastable glissile state similar to those proposed for screw dislocations in HCP metals or intermetallics is shown to be quite unlikely in the present case.

\section{References}

[1] Louchet F., Cochet-muchy D., Brechet Y. and Pelissier J., Phil. Mag. A 57 (1988) 327-335.

[2] HiRTH J.P. and LOTHE H., Theory of Dislocations (New York, Mc Graw Hill, 1968.

[3] LOUCHET F., submitted to Euromat (Paris, June 1993).

[4] PELISSIER J. and LOUChET F., same conference.

[5] FRIEDEL J., Dislocations.

[6] Triki A., Bley F., BReChet Y. and Louchet F., Electron Microsc. Plasticity Fracture Research Mater. Physical Research 14, Eds. U. Messerschmidt, F. Appel, J. Heydenreich and V. Schmidt (Akademie Verlag, Berlin, 1990).

[7] Couret A., Cresatou J., Clement N., Coujou A., Farenc S. and Caillard D., same conference. 